\title{
PERIODONTAL HEALTH STATUS EVALUATION USING CPITN SCORE, IN A GROUP OF PATIENTS WITH RHEUMATOID ARTHRITIS
}

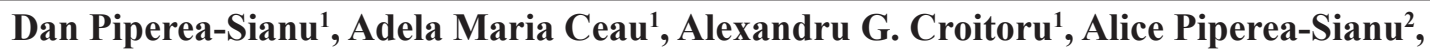 \\ Diana E. Radulescu ${ }^{2}$, Daniela Badita ${ }^{1}$, Sorin Stefan Arama ${ }^{1}$, Carina Mihai ${ }^{1}$ \\ ${ }^{1}$ Carol Davila University of Medicine and Pharmacy, Bucharest \\ ${ }^{2}$ Titu Maiorescu University, Bucharest
}

\begin{abstract}
Introduction. The relationship between periodontal disease (PD) and rheumatoid arthritis (RA) is currently only partially known, both being chronic multifactorial diseases that may share common pathogenic mechanisms and etiological factors. In a previous meta-analysis we concluded that patients with RA are more commonly affected by PD and develop more severe forms of the disease, compared to the general population.

Objectives. The current study aimed to assess the degree of periodontal damage and oral hygiene on a group of patients diagnosed with RA.

Methods. The study was conducted on a group of 30 patients, 6 sextants being assessed in each patient (180 potentially evaluable sextants). Establishing the degree of periodontal damage and oral hygiene was achieved by determining the Community Periodontal Index of Treatment Needs (CPITN) and Oral Hygiene Index (IHB, also known as $\mathrm{OHI})$.

Results. The mean CPITN score/sextant was 1.92. After clinical examination we found 10 edentulous sextants that could not be evaluated. As a result of the distribution of the scores on all the 170 evaluable sextants, we obtained: CPITN $0-11.1 \%$, CPITN $1-23.6 \%$, CPITN $2-32.4 \%$, CPITN $3-27.6 \%$, CPITN $4-5.3 \%$. After analyzing the oral hygiene index the mean value we found was 16. Distribution of IHB intervals was: IHB 0-12 (40\%), IHB 13-24 (53.3\%), IHB 25-36 (6.6\%).

Discussion and conclusions. Comparing our results to data from the literature, in our group of patients with RA the only CPITN index criteria significantly increased, compared to the healthy population, were gingival bleeding on probing and the number of periodontal pockets with 4-5.5 $\mathrm{mm}$ depth.
\end{abstract}

Keywords: periodontal disease, rheumatoid arthritis, CPITN, IHB

\section{INTRODUCTION}

Periodontal disease (PD) is a progressive chronic inflammatory disease, initially affecting the superficial marginal periodontal tissue (gums and supraalveolar ligaments) and subsequently the deep marginal periodontal tissue (root cementum and alveolar bone). The main role in the etiology of PD is awarded to the bacterial plaque, due to the incorporation of periodontal pathogenic agents into its structure. These periodontal pahogenic agents are classified into bacterial groups, which colonize in a specific order, causing the onset and development of marginal periodontal destruction. The determining factor is joined by local predisposing factors, such as tartar, dental cavities, vicious habits, occlusion disorders and general factors: heredity, nutritional deficiencies, systemic disorders, steroid treatments, chemotherapy and antiepileptic drugs etc. In order to clinically objectify the degree of periodontal damage, the following two criteria are generally used: loss of the epithelial insertion (loss of attachment $\mathrm{LOA} \geq 5 \mathrm{~mm}$ ) at two or more sites and the presence of one or more periodontal pockets with a depth $\geq 4$ $\mathrm{mm}$. Long-term effects of periodontal problems are associated with a high prevalence of coronary impairment, independent of the general cardiovascular risk factors. (1-9)

The relationship between PD and rheumatoid arthritis (RA) is currently only partially known, both

Correspondence address:

Dan Piperea-Sianu, PhD student, Faculty Of Dental Medicine, Carol Davila University of Medicine and Pharmacy, 37 Dionisie Lupu Street, Bucharest

E-mail: sianu.dan@gmail.com 
being chronic multifactorial diseases that may share common pathogenic mechanisms and etiological factors. PD may be among the risk factors for acute myocardial infarction, peripheral vascular disease and stroke through atherosclerosis. Thus it is important to know if PD is indeed more prevalent among patients with RA.

In a meta-analysis presented in a previous issue of the Romanian Journal of Reumathology, by the same group of authors, relating to the correlation between PD and RA, we concluded that PD and RA are two chronic inflammatory diseases that can reinforce each other. This association is asserted by epidemiological studies (showing a higher prevalence of periodontal disease in patients with RA and vice versa) and clinical data. Also it was found that an improvement in joint disease follows after treatment of periodontal damage. One of the mechanisms that may explain this association, is represented by the periodontal pathogenic microorganisms that can contribute to increased joint aggression with complex, partially elucidated mechanisms. (10)

\section{OBJECTIVES}

The study aimed to assess the degree of periodontal damage and oral hygiene in a group of patients diagnosed with RA and to identify demographic, socio-economic and clinical factors associated with PD.

\section{MATERIAL AND METHOD}

The study was conducted on a group of 30 patients with RA hospitalized in the Internal Medicine and Rheumatology Clinic of the Dr. Ion Cantacuzino Hospital, Bucharest between March 2013 - January 2014.

Inclusion criteria for the study group were: (a) patients previously diagnosed with RA by the 1987 ACR classification criteria; (b) patients with at least two hospitalizations during this period, in order for the laboratory tests to be conducted and for a brief periodontal examination to be performed.

In order to establish the extent of the periodontal damage and the degree of oral hygiene in patients with RA, the following indices were determined: the Community Periodontal Index of Treatment Needs (CPITN) and the Oral Health Index (IHB).

CPITN belongs to the indices concerning periodontal inflammation and consists in assessing the overall aspect of the gums, the presence of bacterial plaque and tartar, the bleeding of the gingiva during probing, the depth of the periodontal pockets. The periodontal clinical exam is performed on each of the three sextants of each arch.

TABLE 1. CPITN score value - after Croitoru Al. (11)

\begin{tabular}{|l|l|}
\hline \multicolumn{2}{|l|}{ Sextant codes } \\
\hline $\mathbf{0}$ & $\begin{array}{l}\text { Clinically healthy marginal periodontal tissue, on } \\
\text { examination }\end{array}$ \\
\hline $\mathbf{1}$ & Gingival bleeding on probing \\
\hline $\mathbf{2}$ & Supra- and subgingival tartar \\
\hline $\mathbf{3}$ & Periodontal pockets with moderate depth $(4-5.5 \mathrm{~mm})$ \\
\hline $\mathbf{4}$ & Periodontal pockets deeper than $6 \mathrm{~mm}$ \\
\hline
\end{tabular}

IHB belongs to the indices assessing the degree of oral hygiene and the extent of periodontal damage and comprises of two components: the plaque index and the tartar index.

TABLE 2. Plaque and tartar index values for each dental surface - after Croitoru Al. (11)

\begin{tabular}{|l|l|l|l|}
\hline \multicolumn{2}{|l|}{ Plaque index } & \multicolumn{2}{l|}{ Tartar index } \\
\hline $\mathbf{0}$ & Absence of plaque & $\mathbf{0}$ & Absence of tartar \\
$\mathbf{1}$ & $\begin{array}{l}\text { Supragingival plaque, in } \\
\text { the cervical segment }\end{array}$ & $\mathbf{1}$ & $\begin{array}{l}\text { Supragingival tartar, in the } \\
\text { cervical segment }\end{array}$ \\
\hline $\mathbf{2}$ & $\begin{array}{l}\text { Supragingival plaque, in } \\
\text { the mid segment }\end{array}$ & $\mathbf{2}$ & $\begin{array}{l}\text { Supragingival tartar, in the } \\
\text { mid segment }\end{array}$ \\
\hline $\mathbf{3}$ & $\begin{array}{l}\text { Supragingival plaque, } \\
\text { in the occlusal/incisal } \\
\text { segment }\end{array}$ & $\mathbf{3}$ & $\begin{array}{l}\text { Supragingival tartar, in the } \\
\text { occlusal/incisal segment }\end{array}$ \\
\hline
\end{tabular}

The values of plaque and tartar indices are calculated by adding the values found (Table 2) after examining six areas: the buccal surfaces of the right maxillary central incisor and left mandibular central incisor, the buccal surfaces of the first maxillary molars and the oral surfaces of the first mandibular molars.

\section{RESULTS}

The study group included 23 (76.6\%) females. From the distribution by age at the moment of admission, it can be asserverated that the most affected by PD were the patients aged 40-59. Regarding age at the first hospitalization in the study, data are presented in Table 3.

TABLE 3. The distribution of patients in the study group by age in number of cases and percentages

\begin{tabular}{|c|c|c|}
\hline Age group & Number of cases & Percentage \\
\hline $20-29$ & 2 & $6.6 \%$ \\
\hline $30-39$ & 4 & $13.3 \%$ \\
\hline $40-49$ & 10 & $33.3 \%$ \\
\hline $50-59$ & 12 & $40 \%$ \\
\hline $60-70$ & 2 & $6.6 \%$ \\
\hline TOTAL & $\mathbf{3 0}$ & $\mathbf{1 0 0} \%$ \\
\hline
\end{tabular}


Depending on the age at the onset of RA, allocation by age is shown in Table 4.

TABLE 4. The distribution of patients in the study group by age at the clinical onset of $R A$

\begin{tabular}{|c|c|c|}
\hline Age group & Number of cases & Percentage \\
\hline $\mathbf{2 0 - 2 9}$ & 4 & $13.3 \%$ \\
\hline $\mathbf{3 0 - 3 9}$ & 7 & $23.3 \%$ \\
\hline $\mathbf{4 0 - 4 9}$ & 16 & $53.3 \%$ \\
\hline $\mathbf{5 0 - 5 9}$ & 3 & $10 \%$ \\
\hline TOTAL & $\mathbf{3 0}$ & $\mathbf{1 0 0 \%}$ \\
\hline
\end{tabular}

Regarding income, the patients were distributed as follows: (a) people with average income prevailed 17 cases $(56.6 \%)$; (b) 9 people had low-income, representing $30 \%$ of the group. Amongst these the majority was represented by housewives or retired people from rural areas; (c) only 4 people had high incomes, representing $13.3 \%$ of the group. There was a majority of patients from rural areas, with 19 cases (63.3\%), compared to 11 patients from urban areas.

\section{ESTABLISHING THE DEGREE OF PERIODONTAL DAMAGE AND ORAL HYGIENE}

After analyzing the CPITN values, the mean value of the index per sextant was 1.92 .

As the study group included 30 patients and there were potentially 6 evaluable sextants in each patient, we aimed to analyze a maximum of 180 sextants. After clinical examination we identified 10 edentulous sextants that could not be evaluated. The distribution of the remaining 170 sextants on codes was performed as displayed in Table 5.

TABLE 5. Sextant distribution according to code value

\begin{tabular}{|c|c|c|}
\hline Sextant code & Number of sextants & Percentage \\
\hline 0 & 19 & $11.1 \%$ \\
\hline 1 & 40 & $23.6 \%$ \\
\hline 2 & 55 & $32.4 \%$ \\
\hline 3 & 47 & $27.6 \%$ \\
\hline 4 & 9 & $5.3 \%$ \\
\hline Total & $\mathbf{1 7 0}$ & $\mathbf{1 0 0 \%}$ \\
\hline
\end{tabular}

The mean oral hygiene index (IHB) was 16, range 31 .

Patients with RA were distributed in ranges of values (Table 6).

TABLE 6. Distribution of patients according to IHB

\begin{tabular}{|c|c|c|}
\hline Value interval & Number of patients & Percentage \\
\hline $0-12$ & 12 & $40 \%$ \\
\hline $13-24$ & 16 & $53.3 \%$ \\
\hline $25-36$ & 2 & $6.6 \%$ \\
\hline Total & $\mathbf{3 0}$ & $\mathbf{1 0 0 \%}$ \\
\hline
\end{tabular}

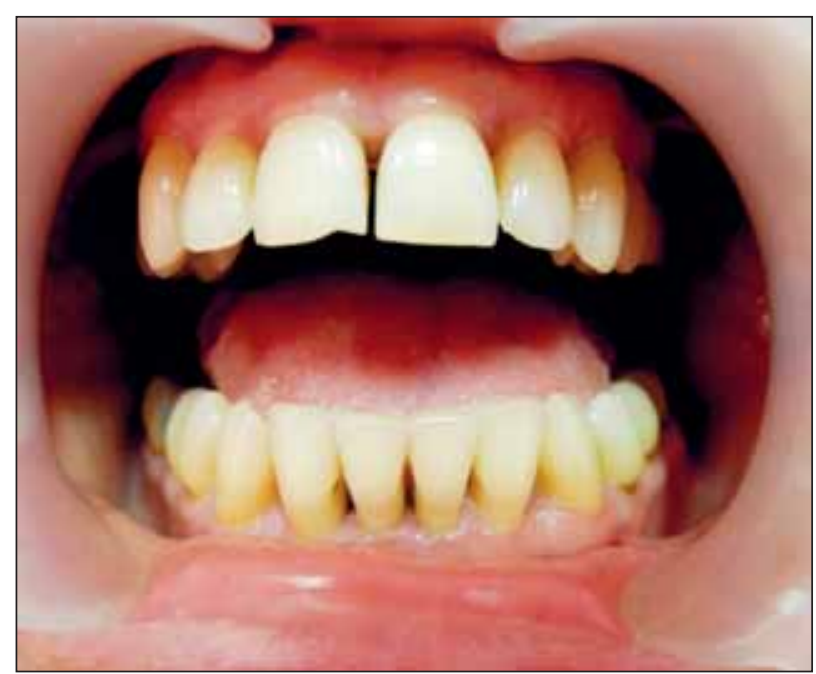

FIGURE 1. Patient with loss of attachment and gingival retractions, with no bleeding on probing, periodontal pockets, plaque or tartar

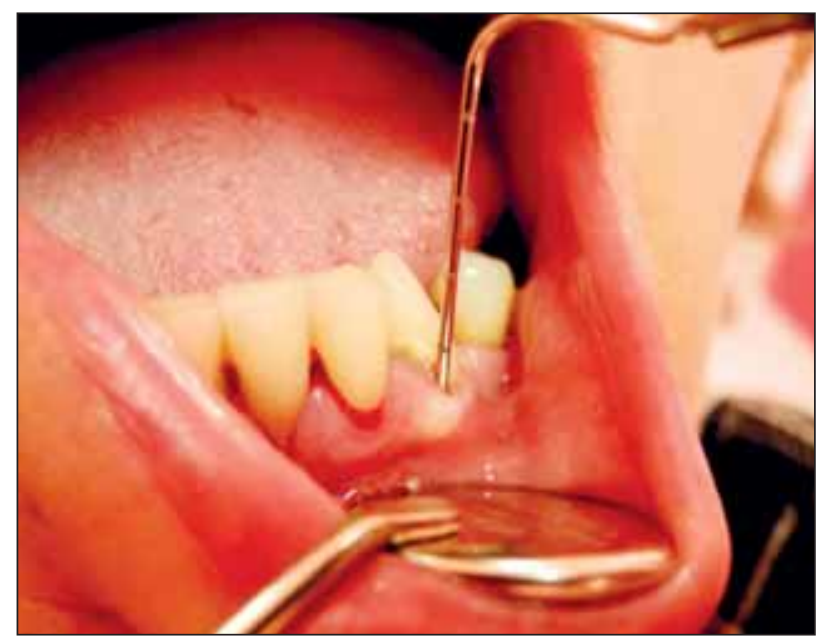

FIGURE 2. Bleeding on probing

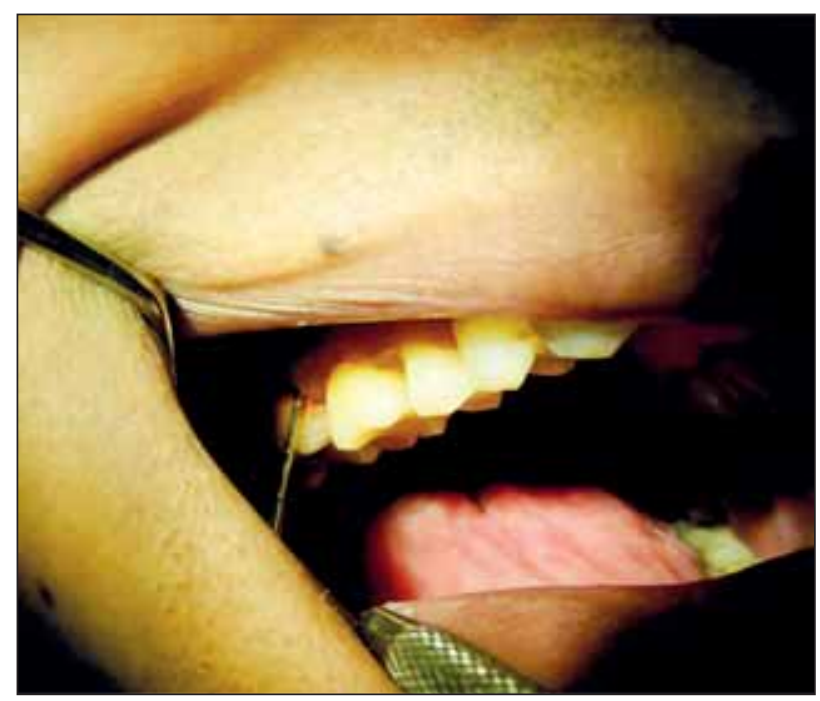

FIGURE 3. Periodontal pocket $\geq 4 \mathrm{~mm}$ 


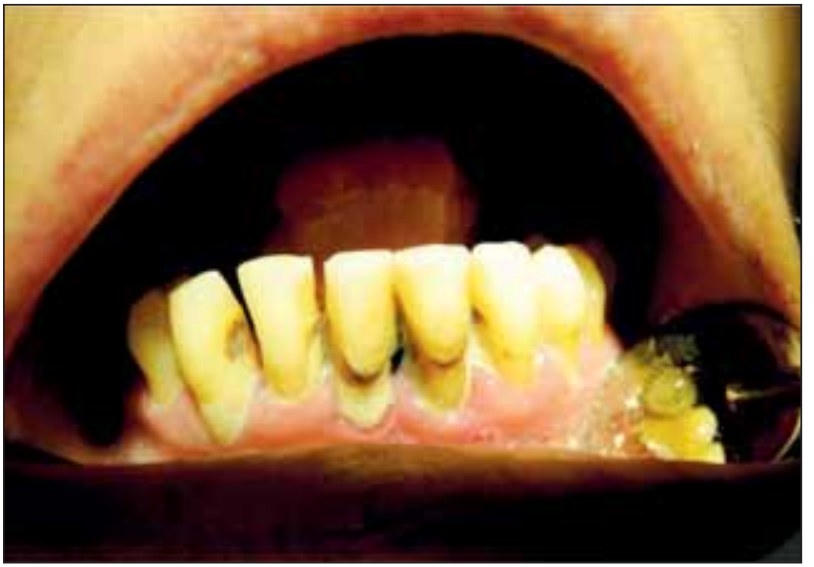

FIGURE 4. Supragingival plaque and tartar, in the cervical segment

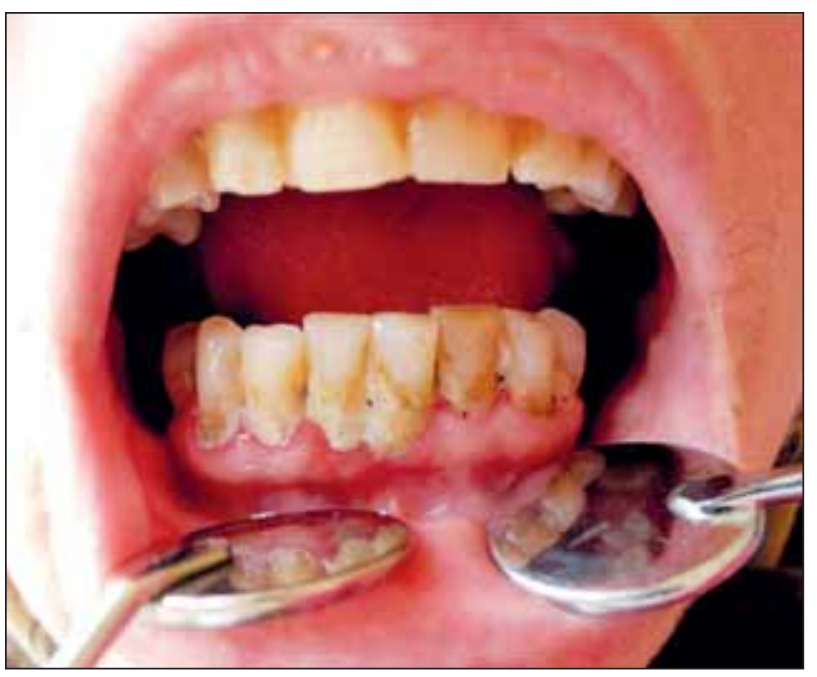

FIGURE 5. Supragingival plaque and tartar, in the mid and cervical segments

\section{DISCUSSIONS AND CONCLUSIONS}

$\mathrm{PD}$ is a pathological complex that brings together multiple forms of periodontal marginal changes, so there is no general consensus on the criteria used for evaluation. In other words, there is a marked heterogenity of criteria for diagnosis and staging that makes comparisons difficult. In this study, we analyzed the degree of periodontal damage and oral hygiene on a group of patients with established RA, using the CPITN index.

CPITN index is widely used, but each group of authors performed statistical reporting differently. We managed to find a few studies reporting the CPITN index for the total number of sextants, in the general population. Following the comparisons, in the group of patients with RA studied by us, the only components of CPITN index significantly increased, as compared to the general population, are bleeding on probing and the number of periodontal pockets with moderate depth $(4-5.5 \mathrm{~mm})$.
Limitations of our study are the small number of patients, at the limit of a uniformly arranged group (30 subjects) and the lack of a control group of ageand gender-matched healthy persons.

In a review presented in a previous issue of the Romanian Journal of Reumathology by our group, analyzing the correlation between PD and RA, epidemiological data and the main mechanisms through which the two diseases influence each other were widely described. The conclusion was that patients with RA are more frequently affected by PD and develop more severe forms of disease. These results are confirmed by the present study. (10)

In our study group, patients aged under 60 years (28 cases, 93.3\%) prevailed, with most patients being aged between 40-59 years (22 cases, 73.3\%). This is not surpirising, RA being a disease with a major impact on the employed, active population. We noticed a large number of patients with onset of rheumatic disease at a young age. A total of 11 patients, accounting for $36.6 \%$ of the group, were diagonsed before the age of 40 .

Distribution of the patients in the study group according to income showed, as expected, the predominance of low and middle-income people. This fact reflects the general distribution of the population in the hospital. Is is widely known that the standard of living has direct implications on the quality of health.

After analyzing the values of CPITN index, a mean code of 1.92 per sextant was found. Regarding the benchmarking of the values obtained in the current study in relation to CPITN code values in the general population, referrals to articles from the literature were performed.

A study conducted in 2014 in Poland, by Konopka et al aimed to evaluate periodontal health, using CPITN score, among the population aged 65-74 years in five cities and four towns. The group included 807 patients, of whom $31.3 \%$ were excluded. The exclusion criteria consisted of total edentulism or presence of a single tooth/sextant. Thus, among patients with enough teeth, a total of 3324 sextants were recorded. CPI codes distribution for the sextans was: $0-1.7 \%, 1-13.7 \%, 2-24.2 \%, 3-31.7 \% 4-28.7 \%$. Relating our results to those of Konopka et al, significant differences can be noticed. In our study group, the percentage of sextants with CPITN code 0 was $11.1 \%$, compared with $1.7 \%$ in the Polish study. Regarding the distribution of value 1 of the CPITN code, notice can be taken over the RA group rate $(23.6 \%)$ that almost doubles the Polish group 
(13.7\%). These differences may be due both to age and income. In the present study, the sextants with CPITN code 0 were seen mainly in young patients, with high-income, from urban areas, concerned with the proper use of various means of oral hygiene and who can regularly carry out dentist appointments, both financially and in terms of the accessibility of health services. The difference between the two groups in terms of age can be taken into account: the study in Poland consisted of elderly patients, thus the degree of PD may be correlated with associated pathology (other than RA), with motor disabilities that make it difficult to travel to dental offices and maintain oral hygiene through brushing or flossing. In our study group, most patients were younger than 60 years, only 2 were within the age range $60-70$ years. CPITN code 2 distribution/sextant was $24.2 \%$ in the Polish study, versus $32.4 \%$ in the group of 30 patients from the present study. Regarding the distribution of CPITN score $4 /$ sextant, the difference between the two studies is important: $28.7 \%$ in the group in Poland, compared with 5.3\% in our group of patients with RA. This difference may be attributed to younger age in our study group, which included patients of various ages, with the majority under 60 years, while the Polish group was composed exclusively of patients over 65 years. Periodontal pockets deeper than $6 \mathrm{~mm}$ (CPITN 4) can be explained by the presence of associated general diseases, and also by periodontal structural changes. These changes happen pathologically but also physiologically due to age, through changes in the qualitative and quantitative properties of saliva that determine decreased immunological defense capacity promoting gingival PD occurrence. (12)

A study published in the Journal of the Indian Society of Periodontology in 2015 aimed to determine the prevalence of PD and the need for periodontal treatment in a group of 500 subjects (295 men, 205 women). The inclusion criteria were: age 15-74 years, the absence of periodontal therapy in the past 6 months, absence of systemic diseases. The value attributed to each sextant was the CPITN maximum code found in the teeth of each sextant. 14 patients were excluded, who were edentulous, not fulfilling the conditions to apply the CPITN index. For the total of 2786 sextants, CPITN code distribution was: CPITN 0 to $15.36 \%$, CPITN 1 to $12.95 \%$ CPITN 2 to $47.2 \%$, CPITN 3 to $12.7 \%$, CPITN 4 to $11.48 \%$. Comparing our patient group to the Indian group, significant differences can be observed. The value 0 of CPITN code was found in a higher percentage of sextants in the Indian group, compared to the current study. The presence of bleeding on probing and deep periodontal pockets of 4 to $5.5 \mathrm{~mm}$ (values 1 and 3 of the CPITN index) were more frequent in the sextants from the group of patients with RA compared to the Indian group without associated diseases. On one hand this would suggest a higher degree of periodontal disease in patients with RA compared to healthy population. On the other hand, plaque and periodontal pockets $\geq 6 \mathrm{~mm}$ (CPITN 2 and CPITN 4) were found more frequently in the sextants from the Asian group. Most likely due to the low number of patients included in our study and due to the fact that most patients $(93.4 \%)$ were aged $<60$ years, the frequency of periodontal pockets $\geq 6 \mathrm{~mm}$ was less frequent than in the Asian group, where their prevalence was favored by the physiological changes of saliva, gingival periodontal structures among elderly patients.(13)

According to the literature, the prevalence of edentulism is higher in RA: RA patients have on average 20 missing teeth, compared with 16 missing teeth in patients without RA. In the study group only 10 edentulous sextants were identified. The low number of edentulous sextants can be explained by the age of the patients and by the small size of the lot. Edentulous sextants were found mainly in people with low income, where the possibility of a conservative treatment can not be considered, thus extraction representing, in most cases, the most accessible method of treatment. $(10,14)$

In conclusion, we found a low level of oral hygiene and a high prevalence of PD in patients with RA, which may be due to several factors. Older age, rural residence, poor education and low income are definitely influencing oral hygiene. Also, the young age at the onset of RA, the chronic course of the disease and the impairing of physical function may increase difficulty in maintaining a rigorous oral hygiene. Therefore, buildup of plaque and tartar are favored, making the patient with RA more prone to PD. On the other hand, chronic infection associated to PD may increase susceptibility to RA, making the relationship between the two diseases even more complex. We believe that the present study is a starting point for further studies, with a larger number of patients and an appropriately selected control group. 


\section{REFERENCES}

1. Croitoru Al., Mihai C., Piperea-Sianu D. et al, Riscul cardiovascular la bolnavii cu poliartrită reumatoidă şi boală parodontală, Revista Română de Reumatologie, 2013; 22(3):129-36.

2. Bretz W.A., Weyant R.J., Corby P.M. et al. Systemic inflammatory markers, periodontal diseases, and periodontal infections in an elderly population, Journal of the American Geriatrics Society, 2005; 53(9):1532-7.

3. Pejcic A., Kesic L.J., Milasin J. Pejcic. C-reactive protein as a systemic marker of inflammation in periodontitis, European Journal of Clinical Microbiology \& Infectious Diseases, 2011; 30(3):407-14.

4. Ramirez-Tortosa M.C., Quiles J.L., Battino M. et al. Periodontitis is associated with altered plasma fatty acids and cardiovascular risk markers, Nutrition, metabolism, and cardiovascular diseases: NMCD, 2010; 20(2):133-9.

5. Buhlin K., Gustafsson A., Pockley A.G. et al. Risk factors for cardiovascular disease in patients with periodontitis, European Heart Journal, 2003; 24(23):2099-107.

6. Dumitriu H.T., Dumitriu S., Dumitriu A.S. Parodontologie, Bucureşti: Editura Viața Medicală Românească, 2009, ISBN: 973-160-027-7.

7. Georgiou T.O., Marshall R.I., Bartold P.M. Prevalence of systemic diseases in Brisbane general and periodontal practice patients, Australian Dental Journal, 2004; 49(4):177-8.
8. Shi D., Meng H., Xu L. et al. Systemic inflammation markers in patients with aggressive periodontitis: a pilot study, Journal of Periodontology, 2008; 79(12):2340-6.

9. Brennan D.S., Spencer A.J, Roberts-Thomson K.F. et al. Periodontal disease among 45-54 year olds in Adelaide South Australia, Australian Dental Journal, 2007; 52:1.

10. Croitoru A.I., Mihai C., Piperea-Şianu D. et al. Implicațiile infecție parodontale la pacienții cu poliartrită reumatoidă, Revista Română de Reumatologie, 2013; 22(2):69-73.

11. Croitoru A.I. Corelații fiziopatologice între boala parodontală, bolile reumatismale autoimune şi riscul cardiovascular, Bucureşti: Editura Cermaprint, 2014, ISBN 978-606-552-139-1.

12. Konopka T., Dembowska E., Pietruska M. et al. Periodontal status and selected parametres of oral condition of Poles Aged from 65 to 74 Years, Przegl Epidemiol, 2015; 69:537-542.

13. Bansal M., Mittal N., Singh T.B. Assessment of the prevalence of periodontal diseases and treatment needs: A hospital-based study, Journal of Indian Society of Periodontology, 2015; 19(2), 211-215.

14. Mercado F.B., Marshall R.I., Klestov A.C. et al. Relationship between rheumatoid arthritis and periodontitis, Journal of Periodontology, 2001; 72:779-787. 